\title{
Eczema Herpeticum in children with burns
}

\author{
Fatima Naumeri ${ }^{1}$, Sushil Rijal ${ }^{2}$, \\ Abdul Rehman Rashid ${ }^{3}$, Hafiz Mahmood Ahmad ${ }^{4}$
}

\begin{abstract}
Background \& Objective: Eczema herpeticum (EH) is a disseminated viral infection occurring in preexisting skin conditions and burns. The objective of this study was to determine the frequency, treatment, and outcome of EH in pediatric burn patients.

Methods: This retrospective study was conducted in the pediatric surgery department, King Edward Medical University/ Mayo Hospital, Lahore, from October 2015 to July 2018 after ethical approval. All pediatric burn patients diagnosed with $\mathrm{EH}$ and not sensitive to Acyclovir or suffering from chemical burns were enrolled in the study. Diagnosis was confirmed by presence of umbilicated lesions in burnt area and a positive Tzanck smear. Intravenous acyclovir and supportive treatment was started. Mortality, development of contractures, length of hospital stay/ time for wound healing, re-activation of EH was calculated.

Results: Out of 3958 admitted pediatric burn patients, 94(2.4\%) developed EH. Girls were 58(61.7\%) and boys were $36(38.3 \%)$. Mean age was $5.16 \pm 2.88$ years. Scald burn was in $43(45.7 \%)$ patients, flame burn in $48(51.1 \%)$ patients, and electric flash burn in $3(3.2 \%)$ patients. Mean TBSA was $21.74 \pm 10.38 \%$. Vesicular eruptions settled in $92(97.9 \%)$ patients after treatment with acyclovir. Mean duration of treatment was $19.89 \pm 8.9$ days and hospital stay was $29.84 \pm 16.98$ days. Twenty three patients $(24.5 \%)$ developed contractures and two patients (2.1\%) developed disseminated EH and expired. Six (6.4\%) patients had reactivation of $\mathrm{EH}$.

Conclusion: EH occurred in 2.4\% of admitted pediatric burn patients. Intravenous acyclovir was successful in $97.9 \%$ of the patients, although $2.1 \%$ developed disseminated EH and expired. Re-activation occurred in $6.4 \%$ of the cases and was associated with prolonged hospital stay.
\end{abstract}

KEYWORDS: Burn, Children, Eczema Herpeticum, Treatment, Incidence, Outcome.

doi: https://doi.org/10.12669/pjms.37.2.3642

How to cite this:

Naumeri F, Rijal S, Rashid AR, Ahmad HM. Eczema Herpeticum in children with burns. Pak J Med Sci. 2021;37(2):421-425. doi: https://doi.org/10.12669/pjms.37.2.3642

This is an Open Access article distributed under the terms of the Creative Commons Attribution License (http://creativecommons.org/licenses/by/3.0), which permits unrestricted use, distribution, and reproduction in any medium, provided the original work is properly cited.

1. Dr. Fatima Naumeri, MCPS, FCPS.

2. Dr. Sushil Rijal, MBBS.

3. Dr. Abdul Rehman Rashid, MBBS

4. Dr. Hafiz Mahmood Ahmad, FCPS

1-4: Department of Pediatric Surgery,

King Edward Medical University/Mayo Hospital,

Lahore, Pakistan.

Correspondence:

Dr. Fatima Naumeri, FCPS, MCPS.

Associate Professor,

Pediatric Surgery Department,

King Edward Medical University,

Lahore, Pakistan.

Email: fatimanaumeri@gmail.com

* Received for Publication:

September 9, 2020

* Revision Received:

* Accepted for Publication:
November 15,2020
October 5,2020

\section{INTRODUCTION}

Eczema Herpeticum, or Kaposi-Juliusberg varicelliform eruption, is a disseminated viral infection characterized by "painful, pruritic umbilicated vesiculopustular eruptions". It commonly occurs in young patients with pre existing skin conditions like atopic dermatitis, burns, and eczema. Patients who are immunecompromised are at a higher risk. This rare disease has widespread morbidity and mortality, associated with bacterial sepsis, viremia and involvement of multiple organs. The exact incidence of disease is not known. In USA annual incidence of hospitalization in children due to 
Eczema Herpeticum (EH) ranges from 4.03 to 7.30 per million. ${ }^{1-3}$

Eczema Herpeticum (EH) is mainly caused by Herpes Simplex Virus (HSV 1) type 1, although Herpes Simplex Virus (HSV) types 2, varicella zoster virus (VZV), cytomegalovirus (CMV) are found in some cases. Patients suffering from EH develop characteristic umbilicated skin eruptions and systemic symptoms like fever, malaise and lymphadenitis. Activation of viral infection like HSV, CMV, and VZV play decisive role in morbidity and mortality in burn patients. ${ }^{1,4}$

The diagnosis of EH is mainly based on clinical examination of skin eruptions and laboratory tests like Tzanck smear, viral culture, direct immunofluorescence, skin biopsy and polymerase chain reaction (PCR). Once the disease is diagnosed, anti-viral drugs are given orally or intravenously with topical application to treat EH. ${ }^{5}$

Limited data is available in literature regarding the incidence and outcome of $\mathrm{EH}$ in pediatric burn patient. However, one study done in United States showed $0.1 \%$ mortality among children admitted in hospital. ${ }^{2}$ Whereas mortality rate is up to $2.7 \%$ in severely burned children with Herpes virus infection. ${ }^{3}$

One study in adult burn patients reported $\mathrm{EH}$ frequency of $4.2 \%$ with mortality rate $11 \% .{ }^{6}$ Although, HSV infection is not straightforwardly associated to higher mortality but it impairs wound healing and may lead to sepsis. ${ }^{4}$ This study was designed to determine the frequency, treatment and outcome of $\mathrm{EH}$ in pediatric burn patients.

\section{METHODS}

This retrospective study was conducted at the department of Pediatric Surgery, King Edward Medical University/ Mayo Hospital, Lahore, from October 2015 to July 2018. Ethical approval was taken from Institutional Review board (No. 532/ RC/KEMU dated 29/07/2020). All burn patients with age less than 13 years and diagnosed with $\mathrm{EH}$ were included, using census sampling technique. Patients who were sensitive to Acyclovir or presented after chemical burns were excluded. Diagnosis of EH was confirmed by a dermatologist on the basis of presence of umbilicated lesions in previously burnt area (Fig.1) and a positive Tzanck smear. For Tzanck smear, scrapping of suspected lesion was done and stained with Giemsa stain. If the smear revealed multinucleated keratinocytes/ giant cells then it was labeled as 'positive'.
Management was started immediately after clinical diagnosis, and intravenous fluids, painkillers, Proton Pump inhibitors and empirical broad spectrum antibiotics (based on ward antibiogram ${ }^{7}$ ) were given initially. Later on culture specific antibiotics were started. Partial healing of lesions and purulent discharge indicating superadded bacterial infection and need of culture specific antibiotics as shown in Fig. 2.

Antiviral injection acyclovir $10 \mathrm{mg} / \mathrm{kg} /$ dose thrice a day was given and continued till complete healing of skin lesions and a negative Tzanck smear. Topically acyclovir and silver sulfadiazine was applied. Tzanck smear was repeatedly every fortnightly to rule out re-activation of $\mathrm{EH}$, once the lesions were settled. Debridement was planned only after negative Tzanck smear and skin grafting was done on subsequent days once wound culture was negative, and wound was healthy. Hemoglobin levels were maintained over $8 \mathrm{gm} / \mathrm{dl}$ during the hospital stay and blood was transfused when required. If patient developed severe allergic reactions to Acyclovir, developed diarrhea or derangement of liver functions, Famciclovir was started.

Variables reviewed were patient characteristics (age, gender, weight, hemoglobin, type of burn, total body surface area [TBSA] involved), co-morbidities such as previous skin disease, features of EH (fever,

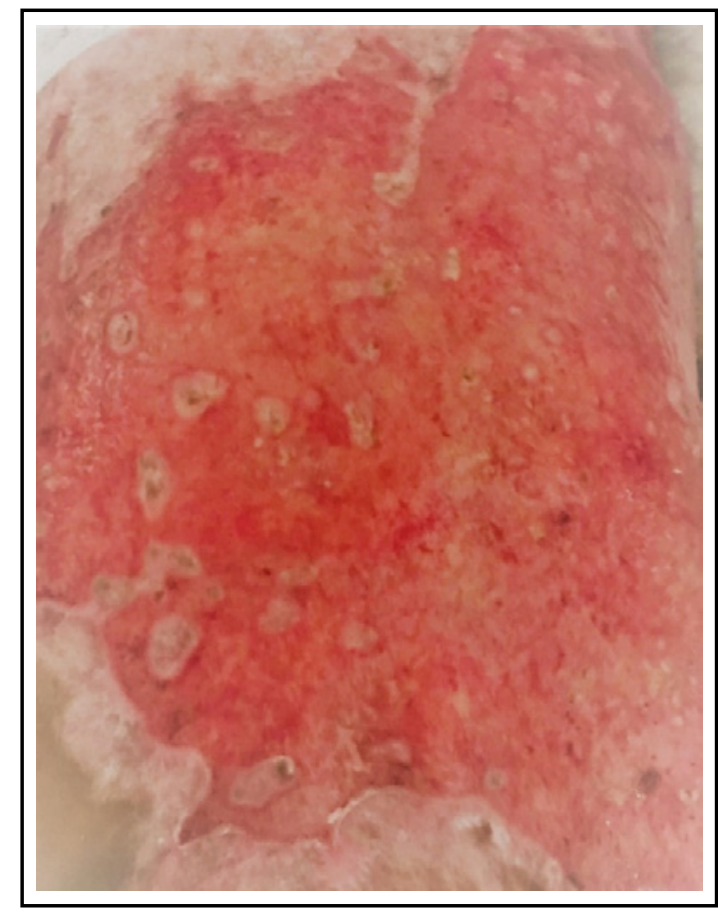

Fig.1: Presence of Umbilicated Lesion of Eczema Herpeticum in Burnt Wound. 


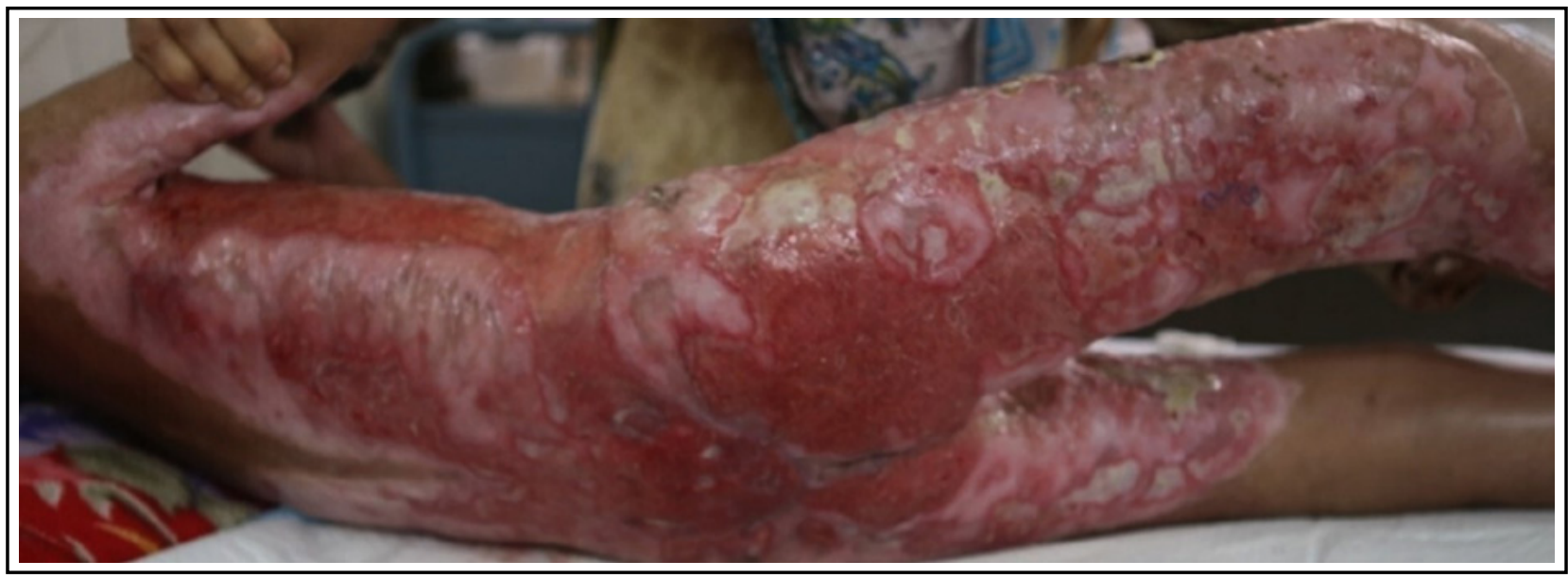

Fig.2: Partially Healed Lesion of Eczema Herpeticum with super added Bacterial infection seen as purulent discharge.

skin eruptions, malaise, lymphadenitis), treatment protocols (duration of acyclovir, healing of eruptions, need of blood transfusions) and outcome (wound healing time, length of hospital stay, contractures, re-activation of $\mathrm{EH}$ and/or mortality).

Data analysis was done by using SPSS 23. Qualitative data (gender, type of burn, TBSA, previous skin disease, fever, malaise, and lymphadenitis, need of transfusion, contractures, re-activation of $\mathrm{EH}$ and mortality) was analyzed as frequency and percentage. Quantitative data (age, weight, hemoglobin, duration of acyclovir, wound healing time, length of hospital stay) was analyzed as mean and standard deviation. Logistic regression was used to analyze association of re-activation of $\mathrm{EH}$ and length of hospital stay, mechanism of burn, TBSA, gender, and age; p-value of $<0.05$ was taken as significant.

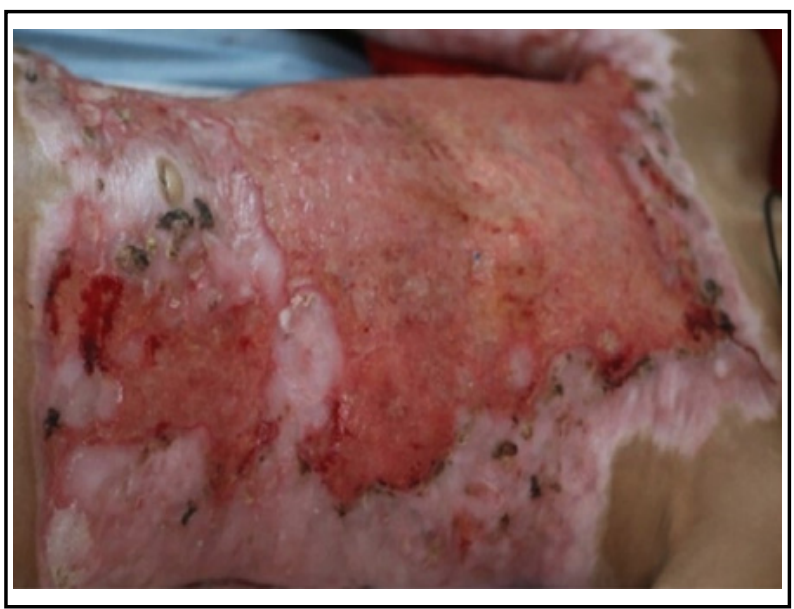

Fig.3: Healed Umbilicated lesions of Eczema Herpeticum.

\section{RESULTS}

Of the 7556 burn patients presenting in pediatric surgical emergency during the study period, 3958 patients were admitted. Ninety four $(2.4 \%)$ patients developed eczema herpeticum (EH) in the admitted burn patients. Of them $58(61.7 \%)$ were female and $36(38.3 \%)$ were male. The mean age of patients was $5.16 \pm 2.88$ years (range 0.33 to12 years). Mean weight of patients was $15.88 \pm 5.78$ kilogram (range 7 to $35 \mathrm{~kg}$ ). The mean hemoglobin was $9.26 \pm 1.86 \mathrm{~g} / \mathrm{dl}$ (range 5.1 to $15.8 \mathrm{~g} / \mathrm{dl}$ ).

Thirty two $(34 \%)$ presented within a day of burn and 62(66\%) presented late. Type of burn was scald burn in $43(45.7 \%)$ patients, flame burn in $48(51.1 \%)$ patients, and electric flash burn in $3(3.2 \%)$ patients.

Mean TBSA was $21.74 \pm 10.38 \%$. (Minimum was $5 \%$ and Maximum was $60 \%)$. All (100\%) of the patients had fever during their hospital stay. Vesicular eruptions settled in 92 (97.9\%) patients after successful treatment with acyclovir. Fig.3 shows healed $\mathrm{EH}$ lesions after antiviral therapy.

Mean duration of treatment was $19.89 \pm 8.9$ days. (Minimum 7 days to Maximum 48 days). Blood transfusion was done in $87(92.6 \%)$ patients and $7(7.4 \%)$ did not require any transfusion during hospital stay. None of the patients gave past history of any skin disease or developed lymphadenitis. No patient developed reaction to Acyclovir.

Mean length of hospital stay was $29.84 \pm 16.98$ days. Out of 94 patients, 23 patients (24.5\%) developed contractures and two patients $(2.1 \%)$ developed disseminated $\mathrm{EH}$ and expired. Six 
(6.4\%) patients had re-activation of $\mathrm{EH}$. There was no association of re-activation of $\mathrm{EH}$ and age ( $p$ value 0.07 ), gender ( $p$ value 0.3 ), TBSA ( $p$ value 0.1 ). Re-activation was associated with longer than one month of hospital stay ( $p$ value $=0.0001$ ).

\section{DISCUSSION}

The frequency of $\mathrm{EH}$ in admitted burn patients in this study was $2.4 \%$. Other studies have shown incidence of $\mathrm{EH}$ is $4.5 \%$ in pediatric population ${ }^{3}$ and $4.2 \%$ in adult population. ${ }^{8} \mathrm{EH}$ can occur in individuals of all ages but is seen more commonly in pediatric patients, especially in children two to three years of age ${ }^{8}$ but in our study mean age is 5.16 years.

Activation of viral infection after burns can occur and lead to eczema herpeticum (EH) in admitted patients. Due to reactivation of HSV, gamma interferon has been prescribed in cases of $\mathrm{EH}$. Though a study conducted by Darji $\mathrm{K}$ et al concluded that gamma interferon have no role in improving EH or preventing its' relapse. ${ }^{9}$

Till now no studies have demonstrated the evaluation of Acyclovir therapy in pediatric burn patients with EH. Although, its use has been described in children hospitalized with $\mathrm{EH}^{3}$ In most cases, acyclovir should be administered intravenously $(1,500 \mathrm{mg} / \mathrm{m} 2$ per day in three divided doses or 15 to $30 \mathrm{mg} / \mathrm{kg}$ per day in three divided doses for seven days) though children who appear well and have limited disease can be treated orally $(1,000$ to $1,200 \mathrm{mg} / \mathrm{d}$ in three to five divided doses for five to seven day). For more severely affected children, acyclovir may be given up to 10 days or at least until no new lesions emerge $^{8}$. In this study, intravenous acyclovir was given till lesions settled. Duration of hospital stay was $29.84 \pm 16.98$ days and duration of acyclovir treatment was $19.89 \pm 8.9$ days. This duration is longer than mean duration of treatment $(9.8 \pm 3.1$ days) mentioned by Luca et al. ${ }^{10}$

In cases of acyclovir resistance, Foscarnet is used. ${ }^{11,12}$ Valacyclovir and Famciclovir are recommended in patients not responding to acyclovir due to their better oral absorption. ${ }^{11-13}$ However Valacyclovir is not recommended in less than 12 years of age. ${ }^{11}$ Our dermatologists advise Famciclovir for any patient who develops allergic reaction to acyclovir, though no patient in this study needed it. Re-activation occurred in $6.4 \%$ cases in this study and needed acyclovir for a further seven days. No association of re-activation could be noted with age, gender, mechanism of burn or TBSA. Re-activation was associated with prolonged hospital stay ( $p$ value 0.0001 ). A study of factors associated with recurrence of $\mathrm{EH}$ revealed recurrence rate of $8.9 \%$ within first month and rate of repeat episode as $16 \%$ after first month, with increased association in hospitalized patients and no association with age, gender, type of treatment or length of hospital stay. ${ }^{10}$ These findings are similar to our study except length of hospital stay.

Additional measures of $\mathrm{EH}$ treatment include local skin care, administration of antipyretic and analgesic medications, provision of other general supportive care, such as intravenous hydration, electrolytes, and blood products. In this study topical acyclovir and silver sulfadiazine was applied. In healed areas topical corticosteroids may be used. Use of corticosteroids topically doesn't affect outcome of EH, while systemic steroids may suppress immunity and lead to dissemination of EH. ${ }^{14,15}$ In this study, generally Hemoglobin levels were maintained at $8 \mathrm{~g} / \mathrm{dl}$ and $92.6 \%$ patients needed blood transfusion.

Appropriate antibiotics should be administered when secondary bacterial infection is suspected. ${ }^{14,16}$ Luca et $\mathrm{al}^{10}$ administered antibiotics in $77.5 \%$ patients who developed secondary infection. In this study all patients needed antibiotic coverage. In a study conducted by Aronson PL et $\mathrm{al}^{17}$ administering empirical antibiotics doesn't affect length of hospital stay except in cases of bacteremia, though earlier acyclovir administration results in earlier discharge ${ }^{16}$. A local study showed that Staphylococcus Aureus is the main pathogen infecting burn patients. ${ }^{18}$ Our ward antibiogram also suggest similar findings, though this study is limited in regards to observing growth patterns in burn patients. ${ }^{7}$

Due to easy availability we preferred using Tzanck smear. More specific investigations include serum herpes simplex virus IgM/IgG levels, transmission electron microscopy for identification of viral particles, PCR for viral DNA, viral culture, and direct fluorescence antibody staining and in atypical cases skin biopsy is required. ${ }^{13,19} \mathrm{In}$ burns, EH may be difficult to differentiate from shingles though lack of involvement of a particular dermatome is helpful in differentiating the two conditions clinically and PCR can provide the definitive diagnosis. ${ }^{20}$

We also isolated the burn patients diagnosed with EH in separate room with separate bath area. Health worker dealing with infected patients were given separate linen, gloves, dressing trolleys/ 
material and hand washing area. For those, who need debridement, sterilized surgical instruments were used in patients and Operation Theater was fumigated after use. Isolated room, beds, chairs, tables, floor and medical instruments used like sphygmomanometer, thermometer, IV stands were routinely decontaminated to prevent from cross infection among other patients, as infection transmission may occur due to direct contact with infected patients' secretions. ${ }^{11}$

Although, HSV infection is not straightforwardly associated with mortality but it impairs wound healing and may lead to sepsis. ${ }^{4}$ Several other studies have revealed that $\mathrm{EH}$ can be widespread and advance to multiple organ failure and critical life-threatening stage. ${ }^{6}$ In this study, two (2.1\%) patients diagnosed with $\mathrm{EH}$ developed disseminated $\mathrm{EH}$ and died. Derek Y Hsu et $\mathrm{al}^{2}$ have mentioned the mortality rate of $0.1 \%$ and it is up to $2.7 \%$ in severely burned children with Herpes virus infection ${ }^{3}$ whereas a study done by Muhammad Sohail and colleagues ${ }^{6}$ in adult population shows mortality rate of $11 \%$. Liaw FY et $\mathrm{al}^{1}$ reported the mortality rate as high as $6 \%$ to $10 \%$ or even $50 \%$ in immune compromised adult population.

Limitations: This is a retrospective study of a single center, and focus mostly on patients residing in Lahore. The other limitation is short follow up of one month only.

\section{CONCLUSION}

$\mathrm{EH}$ occurred in $2.4 \%$ of admitted pediatric burn patients. Intravenous acyclovir was effective in $97.9 \%$ of the patients, although $2.1 \%$ developed disseminated $\mathrm{EH}$ and expired. Re-activation occurred in $6.4 \%$ of the cases and was associated with prolonged hospital stay.

\section{Grant Support \& Financial Disclosures: None.}

\section{REFERENCES}

1. Liaw FY, Huang CF, Hsueh JT, Chiang CP. Eczema herpeticum: a medical emergency. Can Fam Physician. 2012;58(12):1358-1361.

2. Hsu DY, Shinkai K, Silverberg JI. Epidemiology of Eczema Herpeticum in Hospitalized US Children: Analysis of a Nationwide Cohort. J Invest Dermatol. 2018;138(2):265-272. doi: 10.1016/j. jid.2017.08.039

3. Wurzer P, Cole MR, Clayton RP, Hundeshagen G, Lopez ON, Cambiaso-Daniel J, et al. Herpesviradae infections in severely burned children. Burns. 2017;43(5):987-992. doi: 10.1016/j. burns.2017.01.032

4. Wurzer P, Guillory A, Parvizi D, Clayton RP, Branski LK Kamolz LP, et al. Human herpes viruses in burn patients: a systematic review. Burns. 2017;43(1):25-33. doi: 10.1016/j. burns.2016.02.003
5. Siddiqui E, Zia N, Feroze A, Awan S, Ali AL, Razzak JA, et al Burn injury characteristics: findings from Pakistan National Emergency Department Surveillance Study. BMC Emerg Med. 2015;15(Suppl 2):S5. doi: 10.1186/1471-227X-15-S2-S5

6. Sohail M, Khan FA, Shami HB, Bashir MM. Management of eczema herpeticum in a burn unit. J Pak Med Assoc. 2016;66(11):1357-1361.

7. Naumeri F, Mukhtar Z, Sharif M, Rijal S. Comparison of pediatric surgery ward and hospital wide antibiogram in a university hospital. Indo Am J P Sci. 2018;5(11):11388-11395. doi: 10.5281/ zenodo. 1477653

8. Stricker T, Lips U, Sennhauser FH. Visual diagnosis: an 8-monthold infant who has an erupting rash. Pediatr Rev. 2007;28(6):231234. doi: 10.1542/pir.28-6-231

9. Darji K, Frisch S, Adjei Boakye E, Siegfried E. Characterization of children with recurrent eczema herpeticum and response to treatment with interferon-gamma. Pediatr Dermatol. 2017;34(6):686-689. doi: 10.1111/pde.13301

10. Luca NJC, Lara-Corrales I, Pope E. Eczema herpeticum in children: clinical features and factors predictive of hospitalization. J Pediatr. 2012;161(4):671-675. doi: 10.1016/j.jpeds.2012.03.057.

11. Khan A, Shaw L, Bernatoniene J. Fifteen-minute consultation: Eczema herpeticum in a child. Arch Dis Child Educ Pract Ed. 2015;100(2):64-68. doi: 10.1136/archdischild-2013-304460

12. Ferrari B, Taliercio V, Luna P, Abad ME, Larralde M. Kaposi's varicelliform eruption: A case series. Indian Dermatol Online J. 2015;6(6):399-402. doi: 10.4103/2229-5178.169714

13. Zhuang $K, W u Q, \operatorname{Ran} X, \operatorname{Ran} Y$, Ding $L, X u X$, et al. Oral treatment with valacyclovir for HSV-2-associated eczema herpeticum in a 9-month-old infant A case report. Med. 2016;95(29):e4284 doi: 10.1097/MD.0000000000004284

14. Aronson PL, Shah SS, Mohamad Z, Yan AC. Topical corticosteroids and hospital length of stay in children with eczema herpeticum. Pediatr Dermatol. 2013;30(2):215-221. doi: 10.1111/j.15251470.2012.01859.x

15. Sener S, Bayram HG, Karincaoglu Y, Senol M. Kaposi's varicelliform eruption complicating irritant contact dermatitis. Pak J Med Sci. 2012;28(1):225-227.

16. Aronson PL, Yan AC, Mittal MK, Mohamad Z, Shah SS. Delayed acyclovir and outcomes of children hospitalized with eczema herpeticum. Pediatrics. 2011;128(6):1161-1167. doi: 10.1542/ peds.2011-0948

17. Aronson PL, Yan AC, Mohamad Z, Mittal MK, Shah SS. Empiric antibiotics and outcomes of children hospitalized with eczema herpeticum. Pediatr Dermatol. 2013;30(2):207-214. doi: 10.1111/j.1525-1470.2012.01860.x

18. Mehdi SZ, Akber JUD, Nizam M, Dawood K, Buksh AR. Frequency and Antimicrobial Susceptibility Pattern of microorganisms isolated from hospitalized infantile burn cases in a Tertiary Care Hospital. Pak Pediatr J. 2016;40(3):135-142.

19. Karray M, Kwan E, Souissi A. Kaposi Varicelliform Eruption [Updated 2020 Sep 15, accessed on 2020 Sep 30]. In: StatPearls [Internet]. Treasure Island (FL): StatPearls Publishing; 2020 Jan. Available from: https://www.ncbi.nlm.nih.gov/books/ NBK482432/

20. Kubota Y, Kosaka K, Hokazono T, Yamaji Y, Tezuka T, Akita $\mathrm{S}$, et al. Disseminated zoster in an adult patient with extensive burns: A case report. Virol J. 2019;16(68):1-7. doi: 10.1186/ s12985-019-1179-8

\section{Authors Contribution:}

FN: Conceived, prepared manuscript, final approval and is accountable for integrity of the study.

SR: Collected data, wrote synopsis, and approved manuscript.

ARR: Designed, did statistical analysis, and approved manuscript.

HMA: Reviewed, final approval of manuscript. 